\title{
A PRIORI BOUNDS FOR PERIODIC SOLUTIONS OF NEUTRAL DIFFERENTIAL EQUATIONS WITH DELAY
}

\section{\|\|}

\author{
JING-WEN LI AND SUI SUN CHENG
}

\begin{abstract}
A priori bounds are established for periodic solutions of a neutral delay differential equation. Such bounds can be used to establish existence of periodic solutions. The resulting existence results improve some existing ones in the literature.
\end{abstract}

\section{Introduction}

In [1], a priori bounds for periodic solutions of a delay Rayleigh equation of the form

$$
x^{\prime \prime}(t)+\lambda f\left(x^{\prime}(t)\right)+\lambda g(x(t-\tau(t)))=\lambda p(t), \lambda \in(0,1),
$$

are derived. Such bounds are important since standard procedures based on continuation theorems will allow us to imply existence of periodic solutions of

$$
x^{\prime \prime}(t)+f\left(x^{\prime}(t)\right)+g(x(t-\tau(t)))=p(t) .
$$

There are now in the literature many different a priori bounds for periodic solutions of many different differential equations (see e.g. [1-8]). In this paper, we will derive a priori bounds also, but for periodic solutions of a neutral differential equation of the form

$$
x^{\prime \prime}(t)+c x^{\prime \prime}(t-\tau)+\lambda f\left(t, x^{\prime}(t-\omega(t))+\lambda g(t, x(t-\sigma(t)))=\lambda p(t), \lambda \in(0,1), t \in R,\right.
$$

where $\tau$ and $c$ are real numbers such that $|c|<1$. The functions $f, g: R^{2} \rightarrow R$ and $\omega, \sigma, p: R \rightarrow R$ are continuous and there is a $T>0$ such that $f(t+T, u)=f(t)$ and $g(t+T, u)=g(t)$ for $t, u \in R$, and $\omega(t+T)=\omega(t), \sigma(t+T)=\sigma(t)$ as well as $p(t+T)=p(t)$ for $t \in R$. The function $p$ also satisfies the condition

$$
\int_{0}^{T} p(t) d t=0
$$

One reason for deriving such a priori bounds is that we may employ continuation theorems again to show existence of periodic solutions of

$$
x^{\prime \prime}(t)+c x^{\prime \prime}(t-\tau)+f\left(t, x^{\prime}(t-\omega(t))\right)+g(t, x(t-\sigma(t)))=p(t), t \in R .
$$

Received February 22, 2006.

2000 Mathematics Subject Classification. Primary 34K10, 34C25.

Key words and phrases. Neutral delay equation, a priori bound, continuation theorem, periodic solution. 
Another reason is that our a priori bounds are better and more general than the existing ones.

As usual, the maximum norm of a continuous $T$-periodic real function $x=x(t)$ defined on $R$ is denoted by $\|x\|_{0}=\max _{0 \leq t \leq T}|x(t)|$.

Theorem 1. Suppose there is a positive constant $D$ and there are nonnegative constants $K, M$ and $\left.\beta \in[0,2(1-|c|)) / T^{2}\right)$ such that

(i) $|f(t, x)| \leq K$ for $(t, x) \in R^{2}$,

(ii) $x g(t, x)>0$ and $|g(t, x)|>K$ for $t \in R$ and $|x| \geq D$, and

(iii) $g(t, x) \geq-\beta|x|-M$ for $t \in R$ and $x \leq-D$.

Then for any $T$-periodic solution $x$ of (1), we have

and

$$
\|x\|_{0} \leq\left(1-\frac{T^{2} \beta}{2(1-|c|)}\right)^{-1}\left\{D+\frac{T^{2}\left(M+G+K+\frac{1}{2}\|p\|_{0}\right)}{2(1-|c|)}\right\}
$$

$$
\left\|x^{\prime}\right\|_{0} \leq \frac{T}{1-|c|}\left\{\beta\|x\|_{0}+M+G+K+\frac{1}{2}\|p\|_{0}\right\}
$$

where

$$
G=\max _{0 \leq t \leq T,-D \leq x \leq D}|g(t, x)|
$$

Proof. Let $x=x(t)$ be a $T$-periodic solution of (1) (which is necessarily $C^{2}$ ). Let

$$
\begin{aligned}
& A=\{t \in[0, T]: x(t-\sigma(t))>D\} \\
& B=\{t \in[0, T]: x(t-\sigma(t))<-D\} \\
& C=\{t \in[0, T]:|x(t-\sigma(t))| \leq D\}
\end{aligned}
$$

Then

$$
\int_{C}|g(t, x(t-\sigma(t)))| d t \leq T G .
$$

Since $g(t, x)<0$ for $x \leq-D$, we see from condition (iii) that

$$
\begin{aligned}
\int_{B}|g(t, x(t-\sigma(t)))| d t & =\int_{B}-g(t, x(t-\sigma(t))) d t \\
& \leq \int_{B}(\beta|x(t-\sigma(t))|+M) d t \leq T\left(\beta\|x\|_{0}+M\right) .
\end{aligned}
$$

Integrating (1) from 0 to $T$, we see that

$$
\begin{aligned}
0 & =\int_{0}^{T} f\left(t, x^{\prime}(t-\omega(t)) d t+\int_{0}^{T} g(t, x(t-\sigma(t))) d t\right. \\
& =\int_{0}^{T} f\left(t, x^{\prime}(t-\omega(t)) d t+\left\{\int_{A}+\int_{B}+\int_{C}\right\} g(t, x(t-\sigma(t))) d t\right.
\end{aligned}
$$


Hence by conditions (i) and (ii),

$$
\begin{aligned}
\int_{A}|g(t, x(t-\sigma(t)))| d t & =\int_{A} g(t, x(t-\sigma(t))) d t \\
& =-\left\{\int_{B}+\int_{C}\right\} g(t, x(t-\sigma(t))) d t-\int_{0}^{T} f\left(t, x^{\prime}(t-\omega(t)) d t\right. \\
& \leq\left\{\int_{B}+\int_{C}\right\}|g(t, x(t-\sigma(t)))| d t+\int_{0}^{T} \mid f\left(t, x^{\prime}(t-\omega(t)) \mid d t\right. \\
& \leq\left\{\int_{B}+\int_{C}\right\}|g(t, x(t-\sigma(t)))| d t+T K .
\end{aligned}
$$

It follows that

$$
\begin{aligned}
& \int_{0}^{T}|g(t, x(t-\sigma(t)))| d t \\
& \quad \leq 2\left\{\int_{B}+\int_{C}\right\}|g(t, x(t-\sigma(t)))| d t+T K \leq 2 T \beta\|x\|_{0}+2 T M+2 T G+T K .
\end{aligned}
$$

Next, by the periodicity of $x(t)+c x(t-\tau)$, there is $t_{0} \in[0, T]$ such that

$$
x^{\prime}\left(t_{0}\right)+c x^{\prime}\left(t_{0}-\tau\right)=0 .
$$

Thus, integrating (1) from $t_{0}$ to $t \in\left[t_{0}, t_{0}+T\right]$, as well as from $t \in\left[t_{0}, t_{0}+T\right]$ to $t_{0}+T$, we see that

$$
\begin{aligned}
x^{\prime}(t)= & -c x^{\prime}(t-\tau)-\lambda \int_{t_{0}}^{t} f\left(s, x^{\prime}(s-\omega(s))\right) d s-\lambda \int_{t_{0}}^{t} g(s, x(s-\sigma(s))) d s \\
& +\lambda \int_{t_{0}}^{t} p(s) d s
\end{aligned}
$$

and

$$
\begin{aligned}
-x^{\prime}(t)= & c x^{\prime}(t-\tau)-\lambda \int_{t}^{t_{0}+T} f\left(s, x^{\prime}(s-\omega(s))\right) d s-\lambda \int_{t}^{t_{0}+T} g(s, x(t-\sigma(s))) d s \\
& +\lambda \int_{t}^{t_{0}+T} p(s) d s
\end{aligned}
$$

respectively. Applying the triangle inequality, we obtain further that

$$
\left|x^{\prime}(t)\right| \leq|c|\left\|x^{\prime}\right\|_{0}+\int_{t_{0}}^{t}\left|f\left(s, x^{\prime}(s-\omega(s))\right)\right| d s+\int_{t_{0}}^{t}|g(s, x(s-\sigma(s)))| d s+\int_{t_{0}}^{t}|p(s)| d s
$$

and

$$
\begin{aligned}
\left|x^{\prime}(t)\right| \leq & |c|\left\|x^{\prime}\right\|_{0}+\int_{t}^{t_{0}+T}\left|f\left(s, x^{\prime}(s-\omega(s))\right)\right| d s+\int_{t}^{t_{0}+T}|g(s, x(s-\sigma(s)))| d s \\
& +\int_{t}^{t_{0}+T}|p(s)| d s .
\end{aligned}
$$


By adding these inequalities and then applying (7), we may now see that

$$
\begin{aligned}
\left\|x^{\prime}\right\|_{0} & \leq|c|\left\|x^{\prime}\right\|_{0}+\frac{1}{2} \int_{0}^{T}\left|f\left(t, x^{\prime}(t-\omega(t))\right)\right| d t+\frac{1}{2} \int_{0}^{T}|g(t, x(t-\sigma(s)))| d t+\frac{1}{2} \int_{0}^{T}|p(t)| d t \\
& \leq|c|\left\|x^{\prime}\right\|_{0}+\frac{1}{2} T K+\frac{1}{2}\left\{2 T \beta\|x\|_{0}+2 T M+2 T G+T K\right\}+\frac{1}{2} T\|p\|_{0}
\end{aligned}
$$

which implies

$$
\left\|x^{\prime}\right\|_{0} \leq \frac{T}{1-|c|}\left\{\beta\|x\|_{0}+M+G+K+\frac{1}{2}\|p\|_{0}\right\} .
$$

Next, we infer from (6) and the integral mean value theorem that there is some point $\xi \in[0, T]$ such that

$$
f\left(\xi, x^{\prime}(\xi-\omega(\xi))\right)=-g(\xi, x(\xi-\sigma(\xi))) .
$$

Thus, by conditions (i) and (ii), we see that $|x(\xi-\sigma(\xi))| \leq D$. Otherwise

$$
K \geq\left|f\left(\xi, x^{\prime}(\xi-\omega(\xi))\right)\right|=|g(\xi, x(\xi-\sigma(\xi)))|>K
$$

which is a contradiction. Since $x$ is $T$-periodic, there then exists a $t^{*} \in[0, T]$ such that $x\left(t^{*}\right)=x(\xi-\sigma(\xi))$ and

$$
\left|x\left(t^{*}\right)\right|<D
$$

We may now apply an inequality for periodic functions (see [2, Theorem 1]) and obtain

$$
\begin{aligned}
\|x\|_{0} & \leq\left|x\left(t^{*}\right)\right|+\frac{1}{2} \int_{0}^{T}\left|x^{\prime}(t)\right| d t \\
& \leq D+\frac{T}{2}\left\|x^{\prime}\right\|_{0} \leq D+\frac{T^{2}}{2(1-|c|)}\left\{\beta\|x\|_{0}+M+G+K+\frac{1}{2}\|p\|_{0}\right\}
\end{aligned}
$$

which implies

$$
\left(1-\frac{T^{2} \beta}{2(1-|c|)}\right)\|x\|_{0} \leq D+\frac{T^{2}}{2(1-|c|)}\left\{M+G+K+\frac{1}{2}\|p\|_{0}\right\} .
$$

Since $\beta \in\left[0,2(1-|c|) / T^{2}\right)$, we have $T^{2} \beta / 2(1-|c|)<1$ so that (3) is satisfied. This, together with (9), imply $\left\|x^{\prime}\right\|$ satisfies (4). The proof is complete.

There are several variants of Theorem 1.

Theorem 2. Suppose there are positive constants $D, r_{2}$ and nonnegative constants $K, M$ and $r_{1} \in[0,(1-|c|) / T)$ as well as $\beta \in\left[0,\left(\frac{1-|c|}{T}-r_{1}\right) /\left(\frac{r_{1}}{r_{2}}+\frac{T}{2}\right)\right)$ such that

(i) $|f(t, x)| \leq r_{1}|x|+K$ for $(t, x) \in R^{2}$,

(ii) $x g(t, x)>0$ and $|g(t, x)| \geq r_{2}|x|$ for $t \in R$ and $|x| \geq D$, and

(iii) $g(t, x) \geq-\beta|x|-M$ for $t \in R$ and $x \leq-D$. 
Then for any $T$-periodic solution $x$ of (1), we have

$$
\|x\|_{0} \leq\left(1-\left(\frac{r_{1}}{r_{2}}+\frac{T}{2}\right) \frac{T \beta}{1-|c|-r_{1} T}\right)^{-1}\left\{D+\frac{K}{r_{2}}+\left(\frac{T}{2}+\frac{r_{1}}{r_{2}}\right) \frac{T\left(M+G+K+\frac{1}{2}\|p\|_{0}\right)}{1-|c|-r_{1} T}\right\}
$$

and

$$
\left\|x^{\prime}\right\|_{0} \leq \frac{T}{1-|c|-r_{1} T}\left\{\beta\|x\|_{0}+M+G+K+\frac{1}{2}\|p\|_{0}\right\}
$$

where $G$ is defined by (5).

The proof is essentially the same as that of Theorem 1 . We first infer from the new conditions on $f$ and $g$ that

and

$$
\int_{0}^{T}\left|f\left(t, x^{\prime}(t-\omega(t))\right)\right| d t \leq T\left(r_{1}\left\|x^{\prime}\right\|_{0}+K\right)
$$

$$
\int_{0}^{T}|g(t, x(t-\sigma(t)))| d t \leq T\left(2 \beta\|x\|_{0}+r_{1}\left\|x^{\prime}\right\|_{0}+2 M+2 G+K\right) .
$$

Then by following the arguments in the proof of Theorem 1, we may replace the inequality (8) by

$$
\left\|x^{\prime}\right\|_{0} \leq\left(|c|+r_{1} T\right)\left\|x^{\prime}\right\|_{0}+T\left(\beta\|x\|_{0}+M+G+K+\frac{1}{2}\|p\|_{0}\right)
$$

which leads us to (12). Next, we infer from (6) and the integral mean value theorem that there is some point $\xi \in[0, T]$ such that

$$
f\left(\xi, x^{\prime}(\xi-\omega(\xi))\right)=-g(\xi, x(\xi-\sigma(\xi))) .
$$

If $|x(\xi-\tau(\xi))| \leq D$, then

$$
|x(\xi-\tau(\xi))| \leq \frac{r_{1}}{r_{2}}\left\|x^{\prime}\right\|_{0}+D+\frac{K}{r_{2}} .
$$

Otherwise, by conditions (i) and (ii),

$$
K+r_{1}\left|x^{\prime}(\xi-\sigma(\xi))\right| \geq\left|f\left(\xi, x^{\prime}(\xi-\omega(\xi))\right)\right|=|g(\xi, x(\xi-\sigma(\xi)))| \geq r_{2}|x(\xi-\tau(\xi))|,
$$

so that (13) is true. Since $x$ is $T$-periodic, there then exists a $t^{*} \in[0, T]$ such that $x\left(t^{*}\right)=x(\xi-\sigma(\xi))$ and hence

$$
\left|x\left(t^{*}\right)\right| \leq \frac{r_{1}}{r_{2}}\left\|x^{\prime}\right\|_{0}+D+\frac{K}{r_{2}} .
$$

Then

$$
\begin{aligned}
\|x\|_{0} & \leq\left|x\left(t^{*}\right)\right|+\frac{1}{2} \int_{0}^{T}\left|x^{\prime}(t)\right| d t \leq D+\frac{K}{r_{2}}+\left(\frac{T}{2}+\frac{r_{1}}{r_{2}}\right)\left\|x^{\prime}\right\|_{0} \\
& \leq D+\frac{K}{r_{2}}+\left(\frac{T}{2}+\frac{r_{1}}{r_{2}}\right) \frac{T\left(M+G+K+\|p\|_{0} / 2\right)}{1-|c|-r_{1} T}+\left(\frac{r_{1}}{r_{2}}+\frac{T}{2}\right) \frac{T \beta}{1-|c|-r_{1} T}\|x\|_{0} .
\end{aligned}
$$


Invoking the condition on $\beta$, we then see that (11) holds.

Theorem 3. Suppose there are positive constants $D$ and nonnegative constants $K, M$ and $\beta \in\left[0,4(1-|c|) / T^{2}\right)$ such that

(i) $|f(t, x)| \leq K$ for $(t, x) \in R^{2}$,

(ii) $x g(t, x)>0$ and $|g(t, x)|>K$ for $t \in R$ and $|x| \geq D$,

(iii) $|g(t, x)| \leq \beta|x|+M$ for $t \in R$ and $|x| \geq D$.

Then for any $T$-periodic solution $x$ of (1), we have

and

$$
\|x\|_{0} \leq\left(1-\frac{T^{2} \beta}{4(1-|c|)}\right)^{-1}\left\{D+\frac{T^{2}\left(M+G+K+\|p\|_{0}\right.}{4(1-|c|)}\right\}
$$

$$
\left\|x^{\prime}\right\|_{0} \leq \frac{T}{2(1-|c|)}\left\{\beta\|x\|_{0}+M+G+K+\|p\|_{0}\right\}
$$

The proof is essentially the same as that of Theorem 1 . We first infer from the new condition on $g$ that

$$
\int_{0}^{T}|g(t, x(t-\sigma(t)))| d t=\left\{\int_{C}+\int_{[0, T] \backslash C}\right\}|g(t, x(t-\sigma(t)))| d t \leq T G+T\left(\beta\|x\|_{0}+M\right) .
$$

Then by following the arguments in the proof of Theorem 1, we may replace the inequality (9) for $\left\|x^{\prime}\right\|_{0}$ by (15), and replace (10) by

$$
\left|x\left(t^{*}\right)\right| \leq D
$$

for some $t^{*} \in[0, T]$. Then

$$
\begin{aligned}
\|x\|_{0} & \leq\left|x\left(t^{*}\right)\right|+\frac{1}{2} \int_{0}^{T}\left|x^{\prime}(t)\right| d t \leq D+\frac{T}{2}\left\|x^{\prime}\right\|_{0} \\
& \leq D+\frac{T^{2}}{4(1-|c|)}\left\{\beta\|x\|_{0}+M+G+K+\|p\|_{0}\right\} .
\end{aligned}
$$

Invoking the condition on $\beta$, we then see that (14) holds.

Theorem 4. Suppose there are positive constants $D, r_{2}$ and nonnegative constants $K, M$ and $\beta \in\left[0,\left(\frac{2-2|c|}{T}-r_{1}\right) /\left(\frac{r_{1}}{r_{2}}+\frac{T}{2}\right)\right)$ as well as $r_{1} \in\left[0, \frac{2(1-|c|)}{T}\right)$ such that

(i) $|f(t, x)| \leq r_{1}|x|+K$ for $(t, x) \in R^{2}$,

(ii) $x g(t, x)>0$ and $|g(t, x)| \geq r_{2}|x|$ for $t \in R$ and $|x| \geq D$, and

(iii) $g(t, x) \geq-\beta|x|-M$ for $t \in R$ and $x \leq-D$.

Then for any $T$-periodic solution $x$ of (1), we have

$$
\|x\|_{0} \leq\left(1-\left(\frac{r_{1}}{r_{2}}+\frac{T}{2}\right) \frac{T \beta}{2\left(1-|c|-r_{1} T\right.}\right)^{-1}\left\{D+\frac{K}{r_{2}}+\left(\frac{r_{1}}{r_{2}}+\frac{T}{2}\right) \frac{T\left(M+G+2 K+\|p\|_{0}\right.}{2\left(1-|c|-r_{1} T\right)}\right\}
$$

and 


$$
\left\|x^{\prime}\right\|_{0} \leq \frac{T}{2\left(1-|c|-r_{1} T\right)}\left\{\beta\|x\|_{0}+M+G+2 K+\|p\|_{0}\right\} .
$$

We may stay two more results. However, they are the same as Theorem 1 and Theorem 2 respectively, except that condition (iii) is replaced by

(iii') $g(x, t) \leq \beta|x|+M$ for $t \in R$ and $x \geq D$.

We remark if $x=x(t)$ is a bounded $T$-periodic solution of (1), then in view of the periodicity of $g$ (and boundedness of the periodic functions $x$ and $x^{\prime}$ ), we see that there are positive numbers $W_{1}$ and $W_{2}$ such that

$$
|g(t, x(t-\sigma(t)))| \leq W_{1}, t \in R,
$$

and

$$
\left|f\left(t, x^{\prime}(t-\omega(t))\right)\right| \leq W_{2}, t \in R .
$$

By (1), we see that

$$
\left|x^{\prime \prime}(t)\right| \leq|c|\left|x^{\prime \prime}(t-\tau)\right|+\lambda\left|f\left(t, x^{\prime}(t-\omega(t))\right)\right|+\lambda|g(t, x(t-\sigma(t)))|+\lambda|p(t)|, t \in R,
$$

and hence

$$
\left\|x^{\prime \prime}\right\|_{0} \leq(1-|c|)^{-1}\left\{W_{1}+W_{2}+\|p\|_{0}\right\} .
$$

Under the conditions of Theorems 1, 2, 3 and 4, we may thus assert that there are constants $D_{0}, D_{1}$ and $D_{2}$, which are independent of $\lambda$, such that all $T$-periodic solutions of (1) satisfy $\|x\|_{0} \leq D_{0},\left\|x^{\prime}\right\|_{0} \leq D_{1}$ and $\left\|x^{\prime \prime}\right\|_{0} \leq D_{2}$. By standard procedures as explained in various references (see e.g. 2], we may then show that the continuation theorem of Mawhin can be applied to assert that a $T$-periodic solution of (2) exists.

The details will not be repeated here. Instead, we will compare our results with some of the existent ones in the literature. First of all, Theorem 1 in [1] is a special case of our Theorem 1. Next, the main Theorem in [3] is concerned with neutral equations of the form

$$
x^{\prime \prime}(t)+c x^{\prime \prime}(t-\tau)+g(t, x(t-\sigma))=p(t) .
$$

The conditions in it satisfy those in our Theorem 1. In fact, we do not need the Lipschitz condition on $g$ which is required in [3]. Next, Theorem 1 in [4], which is concerned with Duffing equations of the form

$$
x^{\prime \prime}+g(x(t-\tau))=p(t) .
$$

The conditions in it also satisfy those of our Theorem 1. Finally, the conditions in Theorem 3.1 of [5] satisfy the conditions in our Theorem 1 .

\section{References}

[1] G. Q. Wang and S. S. Cheng, A priori bounds for periodic solutions of a delay Rayleigh equation, Appl. Math. Letters 12(1999), 41-44. 
[2] J. W. Li and G. Q. Wang, Sharp inequalities for periodic functions, Appl. Math. E-Notes 5(2005), 75-83.

[3] G. Q. Wang and J. R. Yan, Existence of periodic solutions for second order nonlinear neutral delay equations, Acta Math. Appl. Sinica 47(2004), 379-384 (in Chinese).

[4] X. K. Huang and Z. G. Xiang, The $2 \pi$-periodic solution of Duffing equation $\ddot{x}+g(x(t-\tau))=$ $p(t)$ with delay, Chinese Sci. Bull. 39(1994), 201-203 (in Chinese).

[5] S. W. Ma, Z. C. Wang and J. S. Yu, Periodic solutions of Duffing equations with delay, Diff. Eq. Dynam. Sys. 8(2002), 243-255.

[6] G. Q. Wang and S. S. Cheng, A priori bounds for periodic solutions of a delay Rayleigh equation with damping, Tamkang J. Math. 34(2003), 293-298.

[7] G. Q. Wang and S. S. Cheng, Periodic solutions of nonautonomous delay Rayleigh equations, preprint.

[8] Z. Q. Zhang and Z. C. Wang, Periodic solutions of a class of second order functional differential equations, Acta Math. Appl. Sinica, English Series 21(2005), 95-108.

Department of Mathematics, Shaoyang University, Shaoyang, Hunan 422000, P. R. China. Department of Mathematics, Tsing Hua University, Hsinchu, Taiwan 30043, R. O. China. 